\title{
MinI-REVIEW
}

\section{CUEDC2: an emerging key player in inflammation and tumorigenesis}

\author{
Jianghong Man, Xuemin Zhang ${ }^{\bowtie}$ \\ Institute of Basic Medical Sciences, National Center of Biomedical Analysis, Beijing 100850, China \\ $\triangle$ Correspondence: xmzhang@nic.bmi.ac.cn \\ Received August 2, 2011 Accepted August 14, 2011
}

\begin{abstract}
CUE domain-containing 2 (CUEDC2) is a protein involved in the regulation of the cell cycle, inflammation, and tumorigenesis and is highly expressed in many types of tumors. CUEDC2 is phosphorylated by Cdk1 during mitosis and promotes the release of anaphase-promoting complex or cyclosome (APC/C) from checkpoint inhibition. CUEDC2 is also known to interact with lkB kinase $\alpha$ (IKK $\alpha$ ) and IKK $\beta$ and has an inhibitory role in the activation of transcription factor nuclear factor-KB. Moreover, CUEDC2 plays an important role in downregulating the expression of hormone receptors estrogen receptor$\alpha$ and progesterone receptor, thereby impairing the responsiveness of breast cancer to endocrine therapies. In this review, current knowledge on the multi-functions of CUEDC2 in normal processes and tumorigenesis are discussed and summarized.
\end{abstract}

KEYWORDS CUEDC2, inflammation, cell cycle, nuclear factor- $\mathrm{KB}$, tumorigenesis

\section{INTRODUCTION}

Cancer is a very complicated disease, and its development, also called tumorigenesis, is composed of multiple components and steps. Many elements are believed to contribute to tumorigenesis. For instance, deregulation of cell growth and cell cycle, inhibition of apoptosis, and chronic inflammation have been shown to play important roles in the development of this deadly disease (Hanahan and Weinberg, 2000). Defects in cell cycle regulation, such as mutations of the cell cycle inhibitors $\mathrm{Rb}$ and $\mathrm{p} 53$, also often result in tumorigenesis.

A growing body of evidence suggests that deregulations in the spindle checkpoint, a surveillance mechanism important for the proper segregation of chromosomes during cell division, promote tumorigenesis (Weaver and Cleveland, 2008). Several critical regulators of mitosis, including Mad2, Aurora B, and Securin, have been found to be overexpressed in many types of cancer and are believed to play essential roles in the development of these cancers (Schvartzman et al., 2010).

In last decade, a large amount of evidence has indicated that inflammation contributes to tumorigenesis (Karin, 2006; Mantovani et al., 2008). Many cancers result from chronic inflammation triggered by either extrinsic factors, such as infection, autoimmunity, and tobacco smoke, or intrinsic factors, such as oncogene activation (Aggarwal et al., 2009). Chronic inflammatory responses produce cytokines and growth factors that may promote cell proliferation and suppress apoptosis, and both of these outcomes result in increased risks of cancer. For example, persistent human papillomavirus (HPV) infection is associated with cervical cancer, infections with hepatitis $B(\mathrm{HBV})$ or $\mathrm{C}(\mathrm{HCV})$ viruses increase the risk of hepatocellular carcinoma $(\mathrm{HCC})$, and infections with Epstein-Barr viruses (EBV) are linked to nasopharyngeal cancer and lymphoma. Gastric cancer arises from chronic Helicobacter pylori infection (Karin, 2006; Wu et al., 2009). Although the strong link between inflammation and cancer is generally accepted, the precise mechanistic linkages between them are only beginning to be understood.

CUEDC2, a CUE domain-containing protein, was initially reported to interact with progesterone receptor $(P R)$ and promote progesterone-induced ubiquitination and degradation of receptors (Zhang et al., 2007). The CUE domain, a small ubiquitin-binding motif with approximately 40 amino acid residues, has a dual role in mono- and poly-ubiquitin recognition, as well as in facilitating intramolecular monoubiquitination (Donaldson et al., 2003; Shih et al., 2003). The CUEDC2 protein is ubiquitously expressed in human tissues and organs and is highly expressed in the brain, heart, and 
testis. Recent studies have suggested that CUEDC2 is a multi-functional protein and is involved in the regulation of many key cellular events (Fig. 1), including cell cycle, growth factor signaling, and inflammation (Li et al., 2008; Gao et al., 2011; Pan et al., 2011). More importantly, an increasing amount of research findings suggests that CUEDC2 is an emerging important player in tumorigenesis.

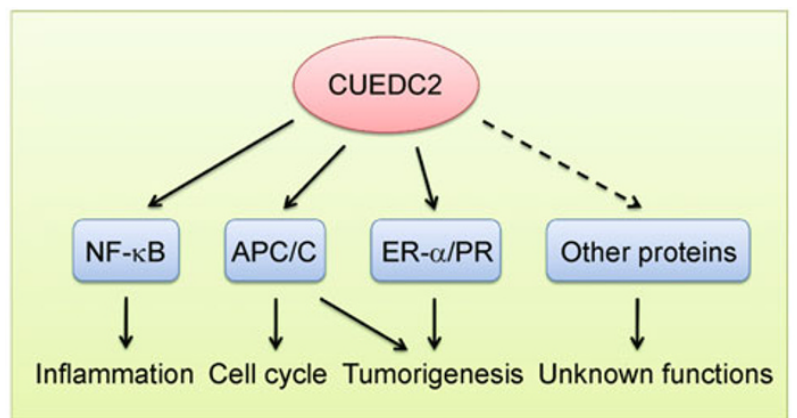

Figure 1. Overview of CUEDC2-mediated signaling pathways and their corresponding biological responses. CUEDC2, CUE domain-containing 2; NF-kB, nuclear factor $\mathrm{KB}$; APC/C, anaphase-promoting complex or cyclosome; ERa/ $\mathrm{PR}$, estrogen receptor $\alpha /$ progesterone receptor.

\section{CUEDC2 AND CELL CYCLE}

The cell cycle is a complicated process by which a single cell develops into a multicellular tissue or even a mature organism. Deregulation of the cell cycle is known to result in various disorders, including tumor development. For instance, mutations of the cell cycle inhibitors $\mathrm{Rb}$ and p53 often result in tumorigenesis. The regulation of cell cycle has been intensively studied. In particular, the fact that the spindle assembly checkpoint (SAC) signal is initiated at unattached kinetochores and that this checkpoint silencing could be achieved by the kinetochore pathway and the release of APC/C from the checkpoint are well established (Weaver and Cleveland, 2008; Schvartzman et al., 2010). However, the underlying molecular mechanisms of the release of APC/C from checkpoint-inhibition after proper kinetochore attachment remain largely unknown.

In a recent study, CUEDC2 was identified as a novel cell cycle regulator that promotes spindle checkpoint inactivation and releases APC/C from checkpoint inhibition (Gao et al., 2011). Mitosis is a complicated and tightly regulated biological process. Ordered protein degradation is known to promote this progression through mitosis (Nigg, 2001; Pines and Rieder, 2001). The primary regulator of protein degradation during mitosis is APC/C, a multi-subunit E3 ubiquitin ligase (Morgan, 1999; Page and Hieter, 1999; Peters, 2006; Sullivan and Morgan, 2007), and which activation in mitosis needs the binding with its co-activator Cdc20 or Cdh1 (Visintin et al., 1997).
CUEDC2 is phosphorylated by Cdk1 in early mitosis, and the depletion of CUEDC2 results in a checkpoint-dependent delay in metaphase-anaphase transition. Moreover, the phosphorylation of CUEDC2 is important for its binding to Cdc20. Interaction between CUEDC2 and Cdc20 is important for the dissociation of Mad2 from the APC/C-Cdc20 complex and subsequently promotes APC/C activation. Similar to other Cdk1 substrates, CUEDC2 is dephosphorylated when cells exit from mitosis. Thus, CUEDC2 is believed to function downstream of Cdk1 to control APC/C activation and metaphase-anaphase transition (Gao et al., 2011).

Identifying mitotic checkpoint complex components and investigating the regulatory events of its activation is very important, and most studies on the regulation of SAC activity have focused on this aspect. A recent study by Gao et al. suggested that the Cdk1-dependent phosphorylation of CUEDC2 is required to turn off SAC. Based on this study, the following model of CUEDC2-mediated regulation of APC/C activation can be established: A dynamic balance exists between the production of Mad2-Cdc20-containing checkpoint complexes and CUEDC2-dependent Mad2 dissociation from Cdc20 during SAC activation. When the rate of Mad2-Cdc20 production exceeds the rate of CUEDC2dependent dissociation, the checkpoint can be established and $A P C / C$ is inhibited. However, when the Mad2-Cdc20 complex is no longer produced as all kinetochores become attached, CUEDC2 promotes Mad2 dissociation from Cdc20 and APC/C activation (Gao et al., 2011).

CUE domains can bind to both monoubiquitin and polyubiquitin, and CUEDC2 has been demonstrated to promote the degradation of PR and ER- $\alpha$ by affecting the ubiquitin-proteasome pathway (Zhang et al., 2007; Pan et al., 2011). Therefore, the assumption that CUEDC2 may be involved in the ubiquitination process during mitosis is an intriguing speculation. In the study by Gao et al., the CUE domain of CUEDC2 was shown to be required for the dissociation of Mad2 from APC/C and for the timely activation of $\mathrm{APC} / \mathrm{C}$. However, to date, no protein degradation mediated directly by CUEDC2 through the ubiquitin system during mitosis has yet been found. These possibilities require further investigation.

\section{CUEDC2 AND NF-KB ACTIVATION/INFLAMMATION}

NF-KB is a transcriptional factor that regulates diverse biological processes, including cell proliferation, survival, inflammation, and immunity (Maniatis, 1999; Karin and BenNeriah, 2000; Chen and Goeddel, 2002; Wajant et al., 2003). Activation of the NF-KB pathway in response to cytokine induction by infection is important for initiating inflammatory responses. The inflammatory response is accurately controlled and terminated as soon as the infection factors are eliminated from the host. These fine regulations of inflammatory responses are achieved through important feedback signaling (Karin and Greten, 2005). Among many known 
regulation steps of NF-KB activation, CUEDC2 acts as an important negative regulator for the pathway and prevents the prolonged activation of NF-KB (Li et al., 2008).

$\mathrm{NF}-\mathrm{KB}$ proteins normally remain inactive through their interaction with their inhibitor, IKB. The regulation of IkB kinase (IKK) activity is a key step in the activation of NF-KB by extracellular stimuli. IKK activity is believed to be controlled by the opposing action of kinases and phosphatases (Yang et al., 2001; Huang et al., 2004; Häcker and Karin, 2006). Before Li et al. reported that CUEDC2 was a major regulator that inactivates IKK, most reports on the NF-KB pathway focused on the mechanisms of IKK activation (Hsu et al., 1996; Nakano et al., 1998). In the study by Li et al., the catalytic IKK subunits IKKa and IKK $\beta$ were both found to interact with CUEDC2 (Li et al., 2008). CUEDC2 was found to inhibit the phosphorylation and activation of IKK $\alpha$ and IKK $\beta$ in response to tumor necrosis factor (TNF) signaling, the degradation of $1 \mathrm{~KB}$ proteins, and the nuclear translocation of NF-KB. CUEDC2 inactivates IKKs by recruiting the protein phosphatase 1 (PP1) to the IKK/ CUEDC2 complex and allowing PP1 to dephosphorylate IKKa and IKKß (Renner and Schmitz, 2009; Bollen et al., 2010). This finding provides another layer for the complexity of $\mathrm{NF}-\mathrm{KB}$ regulation and indicates that IKK is maintained in a default inactive state by its interaction with CUEDC2-PP1, which enables the transient, signal-dependent activation of IKK required for a controlled inflammatory response (Li et al., 2008; Cui et al., 2010).

Receptor-interacting protein (RIP) is well known to play an important role in TNF-induced IKK activation, and TNF receptor associated factor 2 (TRAF2) is partially required because of the redundancy of TRAF5. Studies have indicated that IKK is recruited into the TNFR1 complex through the interaction with TRAF2, and subsequent investigations have suggested that the RIP-IKKY association is also important for the TNF-induced recruitment of IKK. CUEDC2 can interact with IKKa and IKKß and TRAF2 promotes the disassociation of IKK from CUEDC2 (Li et al., 2008). However, the RIP-IKKY complex may also possibly contribute to the displacement of CUEDC2 from IKKs. Therefore, more studies are necessary to investigate the function of RIP in the displacement of IKK from CUEDC2. The interaction of CUEDC2 with ubiquitinated $\mathrm{IKKY}$ is unlikely because IKKY is ubiquitinated by the antiapoptotic protein C-IAP1 (inhibitor of apoptosis protein 1 ), and this process seems to occur in the TNFR1 complex.

The biological and pathogenic importance of NF-kB signaling demonstrates the need to tightly control its action, and its deregulation is often associated with various disorders and diseases, including AIDS, asthma, arthritis, diabetes, stroke, and cancer (Greten et al., 2004; Karin, 2006). As a key regulator of IKK activation, CUEDC2 may affect the pathology of inflammatory diseases and, therefore, be a potential target for the development of new treatments for such disorders and tumors (Minton, 2008).

\section{THE ROLE OF CUEDC2 IN TUMORIGENESIS AND TUMOR THERAPY}

Many factors affect tumorigenesis, including gene mutation and chromosomal instability. Recent studies have demonstrated that CUEDC2 is highly expressed in many types of cancers, including ovarian, kidney, brain, and breast tumors (Gao et al., 2011; Pan et al., 2011). High levels of CUEDC2 result in earlier inactivation of mitotic checkpoints and precocious activation of $\mathrm{APC} / \mathrm{C}$, eventually resulting in chromosome missegregation and aneuploidy (Gao et al., 2011). Aneuploidy can drive tumor formation under certain contexts and is an early event in tumor formation that precedes the inactivation of tumor suppressor genes (Weaver and Cleveland, 2008). Thus, CUEDC2 deregulation may contribute to tumor initiation by causing chromosomal instability. However, the inhibitory role of CUEDC2 in NF-KB activation seems contradictory to its cancer-promoting function as a cell cycle regulator during tumorigenesis. NF-KB signaling pathway is one of the most important pathways involved in inflammation, and inflammation is thought to be a key factor in tumor development, so the inhibitory effect of CUEDC2 on NF-KB activation appears to have a protective effect against tumorigenesis, rather than a promoting one. However, similar to the role of IKK $\beta$ in the HCC model (Maeda et al., 2005), the overexpression of CUEDC2 may promote tumor development by inhibiting NF-KB activation, which results in increased and prolonged c-Jun $\mathrm{N}$-terminal kinase (JNK) activation and enhanced necrotic cell death (Grivennikov et al., 2010). These findings and possibilities provide important insights into the function of CUEDC2 in tumor initiation and promotion.

A recent study suggested that CUEDC2 plays an important role in breast cancer progression and therapy (Pan et al., 2011). Breast cancer is the most common carcinoma in women, and resistance to endocrine therapies is one of the major barriers to its successful treatment. Generally, ER- $\alpha$ expression is the primary biomarker of responses to breast cancer endocrine therapy, and elucidating the regulation of ER- $\alpha$ expression can provide therapeutic targets for overcoming endocrine resistance (Gown, 2008; Yamashita, 2008; Musgrove and Sutherland, 2009).

Pan et al.'s study (2011) further suggested that CUEDC2 interacts with ER- $\alpha$ and downregulates ER- $\alpha$ protein stability through the ubiquitin-proteasome pathway. CUEDC2 expression is inversely correlated with the amount of ER- $\alpha$ protein in breast cancer. In human breast cancer tissues, high levels of CUEDC2 protein are always associated with low ER- $\alpha$ expression, and yield significantly poorer disease-free survival (DFS) and overall survival rates after treatment with tamoxifen. Ectopic CUEDC2 expression has been found to consistently reduce the responsiveness of breast cancer cells to tamoxifen. Therefore, the high expression of CUEDC2 is at 
least partially responsible for the resistance of this subset of breast cancers to endocrine therapy. Approximately $40 \%$ of all ER-a-positive tumors do not respond to tamoxifen therapy despite the recommendation of adjuvant endocrine therapy for people with ER- $\alpha$-positive breast cancer (Normanno et al., 2005). These findings provide an improved understanding of the molecular etiology of tumors that are ER- $\alpha$-positive but also show poor responses to endocrine treatment.

In addition to ER- $\alpha, P R$ and human epidermal growth factor receptor-2 (HER2) are two well-characterized biomarkers of endocrine resistance in breast cancer therapy (Baselga and Norton, 2002; Arpino et al., 2008). Low PR expression or high HER2 expression often indicates poor response to endocrine treatment. Similar to the relationship between CUEDC2 expression and ER- $\alpha$, CUEDC2 and PR expression are also inversely correlated in breast cancer. CUEDC2 binds to PR and promotes PR degradation through the ubiquitinproteasome pathway in breast cancer cells (Zhang et al., 2007). CUEDC2 and HER2 expression are also suggested to have a significant positive correlation in breast cancers, but the mechanism for such remains unknown. Therefore, restoring ER- $\alpha$ expression by inhibiting CUEDC2 provides a potential new strategy for restoring the sensitivity of tumors to endocrine therapy, and CUEDC2 may be a therapeutic target for overcoming endocrine resistance in breast cancers. Future studies are necessary to further explore the role of CUEDC2 deregulation in tumorigenesis.

\section{CONCLUSIONS AND PERSPECTIVES}

The CUEDC2 protein regulates many aspects of cellular events and plays a role in tumorigenesis and tumor response to therapy. CUEDC2 has been demonstrated to have important functions in the cell cycle, steroid receptor regulation, inflammation, and tumorigenesis. Deregulation of the cell cycle and chronic inflammation are known to play pivotal roles in tumor development. As a key regulator of these cellular events, CUEDC2 is warranted to be an emerging important player in tumorigenesis. While further studies on the role of CUEDC2 in tumor development, particularly of different types of cancer, in addition to breast cancer, are necessary, elaboration of the structure of CUEDC2 and modulation of its different functions are also important. These studies will be essential for validating CUEDC2 as an anti-cancer drug target and could provide new strategies for better cancer therapies in the future.

\section{ABBREVIATIONS}

APC/C, anaphase-promoting complex or cyclosome; CUEDC2, CUE domain-containing 2; EBV, Epstein-Barr viruses; HBV or $\mathrm{HCV}$, hepatitis $B$ or $C$ virus; HPV, human papillomavirus; IKK, IkB kinase; NF-KB, nuclear factor-kB; PR, progesterone receptor; RIP, receptorinteracting protein; SAC, spindle assembly checkpoint; TNF, tumor necrosis factor; TRAF2, TNF receptor associated factor 2

\section{REFERENCES}

Aggarwal, B.B., Vijayalekshmi, R.V., and Sung, B. (2009). Targeting inflammatory pathways for prevention and therapy of cancer: short-term friend, long-term foe. Clin Cancer Res 15, 425-430.

Arpino, G., Wiechmann, L., Osborne, C.K., and Schiff, R. (2008). Crosstalk between the estrogen receptor and the HER tyrosine kinase receptor family: molecular mechanism and clinical implications for endocrine therapy resistance. Endocr Rev 29, 217-233.

Baselga, J., and Norton, L. (2002). Focus on breast cancer. Cancer Cell 1, 319-322.

Bollen, M., Peti, W., Ragusa, M.J., and Beullens, M. (2010). The extended PP1 toolkit: designed to create specificity. Trends Biochem Sci 35, 450-458.

Chen, G., and Goeddel, D.V. (2002). TNF-R1 signaling: a beautiful pathway. Science 296, 1634-1635.

Cui, J., Zhu, L., Xia, X., Wang, H.Y., Legras, X., Hong, J., Ji, J., Shen, P., Zheng, S., Chen, Z.J., et al. (2010). NLRC5 negatively regulates the NF-kappaB and type I interferon signaling pathways. Cell 141, 483-496.

Donaldson, K.M., Yin, H., Gekakis, N., Supek, F., and Joazeiro, C.A. (2003). Ubiquitin signals protein trafficking via interaction with a novel ubiquitin binding domain in the membrane fusion regulator, Vps9p. Curr Biol 13, 258-262.

Gao, Y.F., Li, T., Chang, Y., Wang, Y.B., Zhang, W.N., Li, W.H., He, K., Mu, R., Zhen, C., Man, J.H., et al.. (2011). Cdk1-phosphorylated CUEDC2 promotes spindle checkpoint inactivation and chromosomal instability. Nat Cell Biol 13, 924-933.

Gown, A.M. (2008). Current issues in ER and HER2 testing by IHC in breast cancer. Mod Pathol 21, S8-S15.

Greten, F.R., Eckmann, L., Greten, T.F., Park, J.M., Li, Z.W., Egan, L. J., Kagnoff, M.F., and Karin, M. (2004). IKKbeta links inflammation and tumorigenesis in a mouse model of colitis-associated cancer. Cell 118, 285-296.

Grivennikov, S.I., Greten, F.R., and Karin, M. (2010). Immunity, inflammation, and cancer. Cell 140, 883-899.

Häcker, H., and Karin, M. (2006). Regulation and function of IKK and IKK-related kinases. Sci STKE 2006, re13.

Hanahan, D., and Weinberg, R.A. (2000). The hallmarks of cancer. Cell 100, 57-70.

Hsu, H., Huang, J., Shu, H.B., Baichwal, V., and Goeddel, D.V. (1996). TNF-dependent recruitment of the protein kinase RIP to the TNF receptor-1 signaling complex. Immunity 4, 387-396.

Huang, Q., Yang, J., Lin, Y., Walker, C., Cheng, J., Liu, Z.G., and Su, B. (2004). Differential regulation of interleukin 1 receptor and Tolllike receptor signaling by MEKK3. Nat Immunol 5, 98-103.

Karin, M. (2006). Nuclear factor-kappaB in cancer development and progression. Nature 441, 431-436.

Karin, M., and Ben-Neriah, Y. (2000). Phosphorylation meets ubiquitination: the control of NF-[kappa]B activity. Annu Rev Immunol 18, 621-663.

Karin, M., and Greten, F.R. (2005). NF-kappaB: linking inflammation and immunity to cancer development and progression. Nat Rev Immunol 5, 749-759.

Li, H.Y., Liu, H., Wang, C.H., Zhang, J.Y., Man, J.H., Gao, Y.F., Zhang, P.J., Li, W.H., Zhao, J., Pan, X., et al. (2008). Deactivation of the kinase IKK by CUEDC2 through recruitment of the phosphatase PP1. Nat Immunol 9, 533-541. 
Maeda, S., Kamata, H., Luo, J.L., Leffert, H., and Karin, M. (2005). IKKbeta couples hepatocyte death to cytokine-driven compensatory proliferation that promotes chemical hepatocarcinogenesis. Cell 121, 977-990.

Maniatis, T. (1999). A ubiquitin ligase complex essential for the NFkappaB, Wnt/Wingless, and Hedgehog signaling pathways. Genes Dev 13, 505-510.

Mantovani, A., Allavena, P., Sica, A., and Balkwill, F. (2008). Cancerrelated inflammation. Nature 454, 436- 444.

Minton, K. (2008). Stopping before the damage is done. Nat Rev Immunol 8, 372-379.

Morgan, D.O. (1999). Regulation of the APC and the exit from mitosis. Nat Cell Biol 1, E47-E53.

Musgrove, E.A., and Sutherland, R.L. (2009). Biological determinants of endocrine resistance in breast cancer. Nat Rev Cancer 9, 631-643.

Nakano, H., Shindo, M., Sakon, S., Nishinaka, S., Mihara, M., Yagita, H., and Okumura, K. (1998). Differential regulation of IkappaB kinase alpha and beta by two upstream kinases, NF-kappaBinducing kinase and mitogen-activated protein kinase/ERK kinase kinase-1. Proc Natl Acad Sci U S A 95, 3537-3542.

Nigg, E.A. (2001). Mitotic kinases as regulators of cell division and its checkpoints. Nat Rev Mol Cell Biol 2, 21-32.

Normanno, N., Di Maio, M., De Maio, E., De Luca, A., de Matteis, A., Giordano, A., and Perrone, F., and the NCl-Naple Breast Cancer Group. (2005). Mechanisms of endocrine resistance and novel therapeutic strategies in breast cancer. Endocr Relat Cancer 12, 721-747.

Page, A.M., and Hieter, P. (1999). The anaphase-promoting complex: new subunits and regulators. Annu Rev Biochem 68, 583-609.

Pan, X., Zhou, T., Tai, Y.H., Wang, C., Zhao, J., Cao, Y., Chen, Y., Zhang, P.J., Yu, M., Zhen, C., et al. (2011). Elevated expression of CUEDC2 protein confers endocrine resistance in breast cancer. Nat Med 17, 708-714.

Peters, J.M. (2006). The anaphase promoting complex/cyclosome: a machine designed to destroy. Nat Rev Mol Cell Biol 7,
644-656.

Pines, J., and Rieder, C.L. (2001). Re-staging mitosis: a contemporary view of mitotic progression. Nat Cell Biol 3, E3-E6.

Renner, F., and Schmitz, M.L. (2009). Autoregulatory feedback loops terminating the NF-kappaB response. Trends Biochem Sci 34, 128-135.

Schvartzman, J.M., Sotillo, R., and Benezra, R. (2010). Mitotic chromosomal instability and cancer: mouse modelling of the human disease. Nat Rev Cancer 10, 102-115.

Shih, S.C., Prag, G., Francis, S.A., Sutanto, M.A., Hurley, J.H., and Hicke, L. (2003). A ubiquitin-binding motif required for intramolecular monoubiquitylation, the CUE domain. EMBO J 22, 1273-1281.

Sullivan, M., and Morgan, D.O. (2007). Finishing mitosis, one step at a time. Nat Rev Mol Cell Biol 8, 894-903.

Visintin, R., Prinz, S., and Amon, A. (1997). CDC20 and CDH1: a family of substrate-specific activators of APC-dependent proteolysis. Science 278, 460-463.

Wajant, H., Pfizenmaier, K., and Scheurich, P. (2003). Tumor necrosis factor signaling. Cell Death Differ 10, 45-65.

Weaver, B.A., and Cleveland, D.W. (2008). The aneuploidy paradox in cell growth and tumorigenesis. Cancer Cell 14, 431-433.

Wu, S., Rhee, K.J., Albesiano, E., Rabizadeh, S., Wu, X., Yen, H.R., Huso, D.L., Brancati, F.L., Wick, E., McAllister, F., et al. (2009). A human colonic commensal promotes colon tumorigenesis via activation of $\mathrm{T}$ helper type $17 \mathrm{~T}$ cell responses. Nat Med 15, 1016-1022.

Yamashita, H. (2008). Current research topics in endocrine therapy for breast cancer. Int J Clin Oncol 13, 380-383.

Yang, J., Lin, Y., Guo, Z., Cheng, J., Huang, J., Deng, L., Liao, W., Chen, Z., Liu, Z., and Su, B. (2001). The essential role of MEKK3 in TNF-induced NF-kappaB activation. Nat Immunol 2, 620-624.

Zhang, P.J., Zhao, J., Li, H.Y., Man, J.H., He, K., Zhou, T., Pan, X., Li, A.L., Gong, W.L., Jin, B.F., et al. (2007). CUE domain containing 2 regulates degradation of progesterone receptor by ubiquitinproteasome. EMBO J 26, 1831-1842. 